\title{
Determinants in Quantum Matrix Algebras and Integrable Systems
}

\author{
Dimitri Gurevich* \\ Université Polytechnique Hauts-de-France, LMI \\ F-59313 Valenciennes, France \\ and \\ Interdisciplinary Scientific Center J.-V.Poncelet \\ Moscow 119002, Russian Federation \\ Pavel Saponov ${ }^{\dagger}$ \\ National Research University Higher School of Economics, \\ 20 Myasnitskaya Ulitsa, Moscow 101000, Russian Federation \\ and \\ Institute for High Energy Physics, NRC "Kurchatov Institute" \\ Protvino 142281, Russian Federation
}

December 25, 2020

\begin{abstract}
We define quantum determinants in Quantum Matrix Algebras, related to couples of compatible braidings following the scheme from $\mathrm{G}$. We establish relations between these determinants and the so-called column-(row-)determinants, often used in the theory of integrable systems. Also, we generalize the quantum integrable spin systems from [CFRS] by using generalized Yangians, related to couples of compatible braidings. We demonstrate that such quantum integrable spin systems are not uniquely determined by the "quantum coordinate ring" of the basic space $V$. For instance, the "quantum plane" $x y=q y x$ gives rise to two different integrable systems: rational and trigonometric ones.
\end{abstract}

AMS Mathematics Subject Classification, 2010: 81R50, 81R12

Keywords: compatible braidings, quantum matrix algebras, half-quantum algebras, generalized Yangians, quantum symmetric polynomials, quantum determinant

\section{Introduction}

The notion of quantum (or q-)determinant was introduced in the papers of L.D. Faddeev's school (see, for instance, [KS] ) in connection with Quantum Inverse Scattering Method. Initially, such determinants were introduces in RTT algebras, associated with the $U_{q}(\operatorname{sl}(N)) R$-matrices or with some current (i.e. depending on spectral parameters) $R$-matrices.

*gurevich@ihes.fr

${ }^{\dagger}$ Pavel.Saponov@ihep.ru 
Nevertheless, in [G] a large family of other involutive and Hecke symmetries was constructed and quantum determinants were defined in RTT algebras, associated with even 1 symmetries.

Let us recall that these symmetries are particular cases of braidings, that it operators $R$ : $V^{\otimes 2} \rightarrow V^{\otimes 2}$ subject to the so-called braid relation

$$
(R \otimes I)(I \otimes R)(R \otimes I)=(I \otimes R)(R \otimes I)(I \otimes R) .
$$

Hereafter, $V$ is a finite dimensional vector space (called basic) and $I$ stands for the identity operator or matrix 2 .

A braiding $R$ is called a Hecke symmetry (resp., an involutive symmetry), if it meets a supplementary condition

$$
(R-q I)\left(R+q^{-1} I\right)=0, \quad q \neq \pm 1, \quad\left(\text { resp. }, R^{2}=I\right) .
$$

The nonzero parameter $q \in \mathbb{C}$ is assumed to be generic, i.e. such that

$$
k_{q}=\frac{q^{k}-q^{-k}}{q-q^{-1}} \neq 0, \quad \forall k \in \mathbb{Z} .
$$

The objective of the present paper is three-fold. First, by using the scheme from [G], we define quantum determinants in quantum matrix algebras (QMA), associated with couples of compatible braidings introduced in [IOP1], half-quantum algebras (HQA) defined in [IO], and generalized Yangians introduced in [GS1, GS2]. Also, we show that in some RTT algebras (including those associated with the quantum group (QG) $U_{q}(s l(N))$ ) quantum determinants can be cast under the form of column-(or row-)determinants, which are very popular in the literature on integrable systems.

Second, by using quantum elementary symmetric polynomials, closely related to the quantum determinants, we exhibit the Bethe subalgebras in all generalized Yangians in question. Thus, we get quantum integrable systems, which are a far-reaching generalization of the spin systems from [CFRS] and their rational counterparts. Different forms of the corresponding determinants are also discussed.

Third, we would like to draw the reader's attention to the fact that, contrary to a popular belief, the "quantum coordinate ring" of the basic space $V$ does not uniquely determine the corresponding quantum algebra and quantum determinant. Thus, the so-called "quantum plane" defined by $x y-q y x=0$, gives rise to two completely different RTT algebras and generalized Yangians and consequently, leads to two different integrable systems.

The paper is organized as follows. In the next section, by using the method of [G], we define the quantum determinants in the QMA associated with couples $(R, F)$ of compatible braidings, where $R$ is an even involutive or Hecke symmetry. In section 3, we exhibit some properties of the quantum determinants in different algebras. In particular, we describe symmetries which allow defining column-(or row-)determinants. Also, we consider the quantum determinants in the right and left half-quantum algebras. In Section 4 we define the quantum determinant in rational and trigonometric generalized Yangians and exhibit integrable systems associated with such Yangians.

Acknowledgement The authors are indebted to Vladimir Rubtsov for elucidating discussions. The work of P.S. was partially supported by the RFBR grant 19-01-00726-a.

Conflict of Interest: The authors declare that they have no conflicts of interest.

\footnotetext{
${ }^{1}$ The term even means that the $R$-skew-symmetric algebra $\Lambda_{R}(V)$ has a finite number of non-trivial homogenous components and the highest non-trivial component $\Lambda_{R}^{m}(V)$ is one-dimensional. In this case we say that $R$ is of rank $m$.

${ }^{2}$ Note that the operators $P R$ where $P$ is the flip, are subject to the so-called Quantum Yang-Baxter Equation and are usually called $R$-matrices.
} 


\section{Quantum determinants in QMA}

With any Hecke symmetry 3 we associate the $R$-symmetric and $R$-skew-symmetric algebras of the space $V$ defined respectively as the following quotients of the free tensor algebra $T(V)$ of the space $V$ :

$$
\operatorname{Sym}_{R}(V)=T(V) /\langle\operatorname{Im}(q I-R)\rangle, \quad \Lambda_{R}(V)=T(V) /\left\langle\operatorname{Im}\left(q^{-1} I+R\right)\right\rangle .
$$

Here $\langle J\rangle$ stands for a two-sided ideal generated by a set $J \subset T(V)$. The ground field is $\mathbb{C}$.

Each homogenous component $\operatorname{Sym}_{R}^{(k)}(V)$ (resp., $\Lambda_{R}^{(k)}(V)$ ) can be identified with the image of the $R$-symmetrizer $S^{(k)}(R)$ (resp., $R$-skew-symmetrizer $A^{(k)}(R)$ ) acting on the space $V^{\otimes k}$. These projectors can be defined by the following recursion formulae

$$
\begin{array}{ll}
S^{(1)}=I, & S^{(k)}=\frac{1}{k_{q}} S^{(k-1)}\left(q^{-(k-1)} I+(k-1)_{q} R_{k-1 k}\right) S^{(k-1)}, \quad k \geq 2, \\
A^{(1)}=I, & A^{(k)}=\frac{1}{k_{q}} A^{(k-1)}\left(q^{k-1} I-(k-1)_{q} R_{k-1 k}\right) A^{(k-1)}, \quad k \geq 2 .
\end{array}
$$

As usual, the bottom indices indicate the positions where matrices or operators are located. These formulae can be deduced from the representation theory of the symmetric group, provided $R$ is involutive, or that of the Hecke algebra, provided $R$ is a Hecke symmetry (see [Gy]).

Let $R$ and $F$ be braidings. Following [IOP1] we say that the ordered couple $(R, F)$ is compatible (or braidings $R$ and $F$ are compatible) if the following relations take place

$$
R_{12} F_{23} F_{12}=F_{23} F_{12} R_{23}, \quad R_{23} F_{12} F_{23}=F_{12} F_{23} R_{12} \text {. }
$$

Below, we always assume $R$ to be an involutive or a Hecke symmetry.

Let $L=\left\|l_{j}^{i}\right\|_{1 \leq i, j \leq N}$ be an $N \times N$ matrix and $L_{1}=L \otimes I_{2 \ldots p}, p \geq 2$. (Thus, $L_{1}$ is an $N^{p} \times N^{p}$ matrix.) We introduce the following notation:

$$
L_{\overline{1}}=L_{1}, \quad L_{\overline{k+1}}=F_{k k+1} L_{\bar{k}} F_{k k+1}^{-1}, \quad k \leq p-1 .
$$

In the case $F=P$, where $P$ stands for the usual flip, we recover the standard definition: $L_{k+1}=P_{k k+1} L_{k} P_{k k+1}$.

Now, we define a QMA $\mathcal{L}(R, F)$ as a unital associative algebra generated by matrix elements of the matrix $L=\left\|l_{i}^{j}\right\|$ subject to the system of commutation relations:

$$
R_{12} L_{\overline{1}} L_{\overline{2}}=L_{\overline{1}} L_{\overline{2}} R_{12}
$$

The matrix $L$ is called the generating matrix of the algebra $\mathcal{L}(R, F)$.

Observe that the compatibility of the braidings $R$ and $F$ entails that the defining relations of the algebra $\mathcal{L}(R, F)$ can be pushed forward to higher positions in the following sense

$$
R_{k k+1} L_{\bar{k}} L_{\overline{k+1}}=L_{\bar{k}} L_{\overline{k+1}} R_{k k+1}, \quad \forall k<p .
$$

Note that each of the couples $(R, P)$ and $(R, R)$ is evidently compatible. The corresponding algebras $\mathcal{L}(R, P)$ and $\mathcal{L}(R, R)$ are respectively the RTT algebra and Reflection Equation (RE) on 4 . The defining relations of the former algebra $\mathcal{L}(R, P)$ read

$$
R_{12} L_{1} L_{2}=L_{1} L_{2} R_{12} \text {. }
$$

\footnotetext{
${ }^{3}$ In this section we mainly deal with Hecke symmetries. The corresponding results and formulae for involutive symmetries can be obtained by setting $q=1$.

${ }^{4}$ One more example of compatible braidings $(R, F)$ is that formed by braidings from (2.6), where the second matrix plays the role of $R$. Also, an example of such couples is exhibited in IOP2.
} 
The defining relations of the RE algebra $\mathcal{L}(R, R)$ can be cast under the following form:

$$
R_{12} L_{1} R_{12} L_{1}=L_{1} R_{12} L_{1} R_{12} \text {. }
$$

Remark 1 It should be emphasized that if a symmetry $R$ is a deformation of the usual flip the corresponding RTT and RE algebras are deformations of the commutative algebra $\operatorname{Sym}(g l(N))$, i.e. dimensions of the homogenous components of these QMA are classical (if $R$ is a Hecke symmetry, the parameter $q$ must be generic).

However, if $R$ is a braiding coming from the quantum groups of the series $B_{n}, C_{n}, D_{n}$, this property fails. Thus, any similar deformation of the algebra $\operatorname{Sym}(\mathfrak{g})$, where $\mathfrak{g}$ is a Lie algebra belonging to one of these series, does not exist. By contrary, there exist quantum deformations of the function algebra $\operatorname{Fun}(G)$, where $G$ is the corresponding Lie group. The corresponding quotients of the RTT algebras are exhibited in [FRT].

Now, assume the symmetry $R$ to be even. Let $\Lambda_{R}^{(m)}(V), m \geq 2$ be the highest non-trivial homogenous component 5 of the algebra $\Lambda_{R}(V)$. Since, by definition, the dimension of this component is 1 , then there exist two tensors

$$
\mathrm{u}=\left\|u_{i_{1} \ldots i_{m}}\right\| \quad \text { and } \quad \mathrm{v}=\left\|v^{j_{1} \ldots j_{m}}\right\|,
$$

such that

$$
A^{(m)}\left(x_{i_{1}} \otimes \ldots \otimes x_{i_{m}}\right)=u_{i_{1} \ldots i_{m}} v^{j_{1} \ldots j_{m}} x_{j_{1}} \otimes \ldots \otimes x_{j_{m}}, \quad\langle\mathrm{v}, \mathrm{u}\rangle:=v^{i_{1} \ldots i_{m}} u_{i_{1} \ldots i_{m}}=1 .
$$

Hereafter, $\left\{x_{i}\right\}_{1 \leq i \leq N}$ is a basis of the space $V$ and summation over repeated indices is always understood. Thus, the element $v^{j_{1} \ldots j_{m}} x_{j_{1}} \otimes \ldots \otimes x_{j_{m}}$ is a generator of $\operatorname{Im}\left(A^{(m)}\right)$. Note that the tensors $\mathrm{u}$ and $\mathrm{v}$ are defined up to a rescaling 6

$$
\mathrm{u} \mapsto a \mathrm{u}, \quad \mathrm{v} \mapsto a^{-1} \mathrm{v}, \quad a \in \mathbb{C}, a \neq 0 .
$$

By analogy with $[\mathrm{G}]$ we introduce the following definition.

Definition 2 The element of QMA $\mathcal{L}(R, F)$

$$
\operatorname{det}_{\mathcal{L}(R, F)}(L):=\left\langle\mathrm{v}\left|L_{\overline{1}} \ldots L_{\bar{m}}\right| \mathrm{u}\right\rangle:=v^{i_{1} \ldots i_{m}}\left(L_{\overline{1}} \ldots L_{\bar{m}}\right)_{i_{1} \ldots i_{m}}^{j_{1} \ldots j_{m}} u_{j_{1} \ldots j_{m}}
$$

is called the quantum determinant of the generating matrix $L$.

Of course, the quantum determinant $\operatorname{det}_{\mathcal{L}(R, F)}(L)$ can be written in other explicit forms, modulo the defining relations of the QMA $\mathcal{L}(R, F)$. Some of them are presented below.

The form (2.5) will be called canonical.

Consider now two examples. Let us fix a basis $\left\{x=x_{1}, y=x_{2}\right\}$ of the basic space $V$, $N=\operatorname{dim} V=2$ and introduce two symmetries, represented by the following matrices in this basis

$$
\left(\begin{array}{cccc}
1 & 0 & 0 & 0 \\
0 & 0 & q & 0 \\
0 & q^{-1} & 0 & 0 \\
0 & 0 & 0 & 1
\end{array}\right), \quad\left(\begin{array}{cccc}
q & 0 & 0 & 0 \\
0 & q-q^{-1} & 1 & 0 \\
0 & 1 & 0 & 0 \\
0 & 0 & 0 & q
\end{array}\right) .
$$

Each of these symmetries is a deformation of the usual flip $P$. The former symmetry is involutive, the latter one is a Hecke symmetry, coming from the QG $U_{q}(s l(2))$.

\footnotetext{
${ }^{5}$ In general, $m$ could be different from $N=\operatorname{dim} V$ (see G, GPS2]).

${ }^{6}$ If the rank of a symmetry $R$ equals 2 , it is possible to recover the symmetry $R$ by knowing $\mathrm{u}$ and $\mathrm{v}$. All couples $(\mathrm{u}, \mathrm{v})$, giving rise to such symmetries are classified in $\mathrm{G}$.
} 
For the involutive symmetry we have

$$
\mathrm{u}=\left(u_{11}, u_{12}, u_{21}, u_{22}\right)=\frac{1}{2}\left(0,1,-q^{-1}, 0\right), \quad \mathrm{v}=\left(v^{11}, v^{12}, v^{21}, v^{22}\right)=(0,1,-q, 0) .
$$

For the Hecke symmetry we have

$$
\mathrm{u}=\frac{q^{-1}}{2_{q}}(0,1,-q, 0), \quad \mathrm{v}=(0,1,-q, 0)
$$

Note that the tensors $\mathrm{v}$ corresponding to these symmetries coincide with each other and, consequently, the algebras

$$
\operatorname{Sym}_{R}(V)=T(V) /\langle\mathrm{v}\rangle=T(V) /\langle x y-q y x\rangle,
$$

called the "quantum plane", are the same for the both symmetries exhibited in (2.6). Nevertheless, the tensors $\mathrm{u}$ are different. Consequently, the canonical forms of the corresponding determinants $\operatorname{det}_{\mathcal{L}(R, F)}(L)$ differ from each other for all couples $(R, F)$.

Let us compute these determinants for the corresponding RTT algebras $\mathcal{L}(R, P)$ and for the $\mathrm{RE}$ algebras $\mathcal{L}(R, R)$. Denote entries of the generating matrix $L$ of these algebras as follows $l_{1}^{1}=a, l_{1}^{2}=b, l_{2}^{1}=c$ and $l_{2}^{2}=d$ :

$$
L=\left(\begin{array}{ll}
a & b \\
c & d
\end{array}\right) .
$$

Example 1. The defining relations of the RTT algebra $\mathcal{L}(R, P)$, corresponding to the first (involutive) matrix from (2.6)

$$
R_{12} L_{1} L_{2}=L_{1} L_{2} R_{12}
$$

lead to the following systems for generators:

$$
a b=q^{-1} b a, \quad a c=q c a, \quad a d=d a, \quad b c=q^{2} c b, \quad b d=q d b, \quad c d=q^{-1} d c .
$$

According to our definition, the canonical form of the quantum determinant in this algebra is

$$
\operatorname{det}_{\mathcal{L}(R, P)}(L)=\frac{a d+d a}{2}-\frac{q^{-1} b c+q c b}{2} .
$$

With the use of the above commutation relations on the generators the canonical form can be transformed to the following expressions:

$$
\operatorname{det}_{\mathcal{L}(R, P)}(L)=a d-q c b=a d-q^{-1} b c .
$$

The defining relations between the generators of the algebra corresponding to the second matrix from (2.6) are (see [FRT]

$$
a b=q b a, \quad a c=q c a, \quad a d-d a=\left(q-q^{-1}\right) b c, \quad b c=c b, \quad b d=q d b, \quad c d=q d c .
$$

The corresponding quantum determinant is

$$
\operatorname{det}_{\mathcal{L}(R, P)}(L)=\frac{q^{-1} a d+q d a}{2_{q}}-\frac{b c+c b}{2_{q}}=a d-q c b=a d-q b c .
$$

The first expression in (2.10) is the canonical form of the determinant. Other expressions will be discussed in the next section.

Let us also exhibit the corresponding algebras $\Lambda_{R}(V)$. If $R$ is the first symmetry from (2.6), then we have

$$
\Lambda_{R}(V)=T(V) /\left\langle x^{2}, y^{2}, x y+q y x\right\rangle
$$


If $R$ is the second symmetry from (2.6), then the last generator of the ideal in the above quotient should be $q x y+y x$.

Thus, we see that the quantum plane (2.7) gives rise to two different RTT algebras and consequently to two different determinants though we can find a specific form $a d-q c b$ which is the same for both determinants. In the next section we consider higher-dimensional analogs of these algebras and determinants in more details and explain this coincidence.

Example 2. The defining relations

$$
R_{12} L_{1} R_{12} L_{1}=L_{1} R_{12} L_{1} R_{12}
$$

of the $\mathrm{RE}$ algebra $\mathcal{L}(R, R)$, corresponding to the first (involutive) matrix from (2.6), in the explicit form read

$$
a b=b a, \quad a c=c a, \quad a d=d a, \quad b c=c b, \quad b d=d b, \quad c d=d c .
$$

Thus, this algebra is commutative.

It is not a surprising result since the involutive $R$ in $(2.6)$ is connected with the usual flip $P$ by the conjugation

$$
R_{12}=D_{1} P_{12} D_{1}^{-1}, \quad D=\left(\begin{array}{cc}
q^{1 / 2} & 0 \\
0 & q^{-1 / 2}
\end{array}\right) .
$$

Therefore, the matrix

$$
\tilde{L}=D^{-1} L D=\left(\begin{array}{cc}
a & b / q \\
q c & d
\end{array}\right)
$$

satisfies the relation

$$
P_{12} \tilde{L}_{1} P_{12} \tilde{L}_{1}=\tilde{L}_{1} P_{12} \tilde{L}_{1} P_{12} \quad \Leftrightarrow \quad \tilde{L}_{2} \tilde{L}_{1}=\tilde{L}_{1} \tilde{L}_{2}
$$

which means that the entries of $\tilde{L}$ generate a commutative algebra. Therefore, the entries of the matrix $L$ also commute with each other.

The canonical form of the determinant in this case reads

$$
\operatorname{det}_{\mathcal{L}(R, R)}(L)=\frac{a d+d a}{2}-\frac{b c+c b}{2},
$$

or, taking into account the commutativity of the $\mathrm{RE} \mathcal{L}(R, R)$, it can be reduced to the classical expression $a d-b c$.

At last, if we take the second (Hecke) symmetry $R$ from (2.6), we get the following system on the generators

$$
\begin{array}{lll}
q^{2} a b=b a & q^{2} c a=a c & a d=d a \\
q(b c-c b)=\lambda a(d-a) & q(c d-d c)=\lambda c a & q(d b-b d)=\lambda a b,
\end{array}
$$

where we denoted $\lambda=q-q^{-1}$. The canonical form of the determinant is as follows

$$
\operatorname{det}_{\mathcal{L}(R, R)}(L)=\frac{q(a d+d a)}{2_{q}}-\frac{q\left(b c+q^{2} c b\right)}{2_{q}}-\frac{\lambda a^{2}}{2_{q}} .
$$

Upon taking into account the relations between the generators, we can transform the canonical determinant to equivalent forms:

$$
\operatorname{det}_{\mathcal{L}(R, R)}(L)=a d-q^{2} c b=q^{2}(a d-b c)-q \lambda a^{2} .
$$


Another way of introducing quantum analogs of the determinant is based on the notion of the quantum elementary symmetric polynomials defined via the quantum traces. Such a quantum trace is well known in the cases related to the QG $U_{q}(s l(N))$. Nevertheless, the quantum trace can be associated with any skew-invertible braiding $R$ by means of the following method belonging to V.Lyubashenko [L1, L2].

We say that a given braiding $R: V^{\otimes 2} \rightarrow V^{\otimes 2}$ is skew-invertible if there exists an operator $\Psi: V^{\otimes 2} \rightarrow V^{\otimes 2}$ such that

$$
\operatorname{Tr}_{(2)} R_{12} \Psi_{23}=P_{13}=\operatorname{Tr}_{(2)} \Psi_{12} R_{23} \quad \Leftrightarrow \quad R_{i j}^{k l} \Psi_{l m}^{j n}=\delta_{m}^{k} \delta_{i}^{n}=\Psi_{i j}^{k l} R_{l m}^{j n} .
$$

If $R$ is a skew-invertible braiding, the corresponding $R$-trace $\operatorname{Tr}_{R}$ is defined by the formula

$$
\operatorname{Tr}_{R} X=\operatorname{Tr}\left(C^{R} X\right), \quad C^{R}:=\operatorname{Tr}_{(2)} \Psi .
$$

Here $X$ is an arbitrary $N \times N$ matrix (may be with noncommutative entries).

Consider a compatible couple of braidings $(R, F)$ and suppose the braiding $R$ to be skewinvertible. Now, we define a quantum version of the elementary symmetric polynomials in the algebra $\mathcal{L}(R, F)$ as follows

$$
e_{0}=1, \quad e_{k}=\operatorname{Tr}_{R(1 \ldots k)} A^{(k)} L_{\overline{1}} L_{\overline{2}} \ldots L_{\bar{k}}, \quad k \geq 1 .
$$

Hereafter, $\operatorname{Tr}_{(1 \ldots k)}=\operatorname{Tr}_{(1)} \ldots \operatorname{Tr}_{(k)}$.

By using the equality

$$
A^{(k)} L_{\overline{1}} \ldots L_{\bar{k}}=A^{(k)} L_{\overline{1}} \ldots L_{\bar{k}} A^{(k)},
$$

valid in any QMA, we get the following relation

$$
\operatorname{Tr}_{R(1 \ldots m)} A^{(m)} L_{\overline{1}} \ldots L_{\bar{m}}=\operatorname{Tr}_{R(1 \ldots m)} A^{(m)} L_{\overline{1}} \ldots L_{\bar{m}} A^{(m)}=(\mathrm{v} \cdot R \mathrm{u})\left\langle\mathrm{v}\left|L_{\overline{1}} \ldots L_{\bar{m}}\right| \mathrm{u}\right\rangle,
$$

where

$$
(\mathrm{v} \cdot R \mathrm{u})=v^{j_{1} \ldots j_{m}}\left(C^{R}\right)_{j_{1}}^{i_{1}}\left(C^{R}\right)_{j_{2}}^{i_{2} \ldots}\left(C^{R}\right)_{j_{m}}^{i_{m}} u_{i_{1} \ldots i_{m}} .
$$

Thus, the highest elementary symmetric polynomial $e_{m}$ differs from the quantum determinant $\operatorname{det}_{\mathcal{L}(R, F)}(L)$ by a numerical factor. In the particular case $F=P$ these elements are just equal to each other since in this case $(\mathrm{v} \cdot P \mathrm{u})=1$ (note that $C^{P}=I$ ).

\section{Some properties of quantum determinants}

In this section we consider two questions. The first one is: what is the relation between the determinant $\operatorname{det}_{\mathcal{L}(R, F)}(L)$ and the characteristic polynomial of the matrix $L$ ? The second question is: whether the quantum determinant is central? We always assume the rank of a symmetry $R$ to be $m$.

We say that a monic polynomial $c h(t)$ of degree $m$ is characteristic if $c h(L)=0$. In virtue of the Cayley-Hamilton theorem in the classical case $R=F=P$ (the corresponding algebra $\mathcal{L}(P, P)$ is commutative) the characteristic polynomial reads

$$
\operatorname{ch}(t)=\operatorname{det}_{\mathcal{L}(P, P)}(L-t I) .
$$

Proposition 3 In the algebras $\mathcal{L}(R, R)$, where $R$ is a Hecke symmetry, the following relation holds

$$
\operatorname{det}_{\mathcal{L}(R, R)}(L-t I)=\sum_{0 \leq k \leq m}(-t)^{m-k} \alpha_{k} e_{k}
$$

where $\alpha_{k}=q^{m k} \frac{m !}{k !(m-k) !} \frac{k_{q} !(m-k)_{q} !}{m_{q} !}$. 
Proof. For any even Hecke symmetry of the rank $m$ the quantity (2.16) reads

$$
(v \cdot R u)=q^{-m^{2}}
$$

This follows from the relation (see GS2])

$$
\operatorname{Tr}_{R(k+1 \ldots m)} A_{1 \ldots m}^{(m)}=q^{-m(m-k)} \frac{k_{q} !(m-k)_{q} !}{m_{q} !} A_{1 \ldots k}^{(k)}
$$

for $k=0$.

Now, in the expansion of the element

$$
q^{m^{2}} \operatorname{det}_{\mathcal{L}(R, R)}(L-t I)=\operatorname{Tr}_{R(1 \ldots m)} A_{1 \ldots m}^{(m)}(L-t I)_{\overline{1} \ldots}(L-t I)_{\bar{m}}
$$

we put together the terms containing $k$ factors $L_{\bar{i}}$ on some places and the identity matrices on other positions. The number of such terms is $\frac{m !}{k !(m-k) !}$ and they are equal to each other. This property is due to the fact that 7

$$
\operatorname{Tr}_{R(1 \ldots m)} A_{1 \ldots m}^{(m)} L_{\bar{i}_{1}} L_{\bar{i}_{2}} \ldots L_{\bar{i}_{k}}=\operatorname{Tr}_{R(1 \ldots m)} A_{1 \ldots m}^{(m)} L_{\overline{1}} L_{\overline{2}} \ldots L_{\bar{k}}
$$

for any ordered subset of indices $1 \leq i_{1}<i_{2}<\ldots<i_{k} \leq m$. Now, it suffices to apply formula (3.2). Finally, we find

$$
\operatorname{Tr}_{R(1 \ldots m)} A_{1 \ldots m}^{(m)} L_{\overline{1}} \ldots L_{\bar{k}}=q^{-m(m-k)} \frac{k_{q} !(m-k)_{q} !}{m_{q} !} e_{k}(L) .
$$

The proof is completed.

If $R$ is an involutive symmetry, then by setting $q=1$ in (3.1) we get the following claim.

Proposition 4 If $R$ is an involutive symmetry, the polynomial $\operatorname{det}_{\mathcal{L}(R, R)}(L-t I)$ is characteristic.

If $R$ is a Hecke symmetry, the polynomial $\operatorname{det}_{\mathcal{L}(R, R)}(L-t I)$ is not characteristic. However, we get the characteristic polynomial upon replacing $\alpha_{k}$ by $q^{k}$ in the right hand side of (3.1), that is we have:

$$
\operatorname{ch}(t):=t^{m}-q e_{1} t^{m-1}+q^{2} e_{2} t^{m-2}+\ldots+(-q)^{m-1} e_{m-1} t+(-q)^{m} e_{m} .
$$

Thus, upon substituting $t=L$ in this polynomial, we get the Cayley-Hamilton identity for the matrix $L$ :

$$
L^{m}-q e_{1} L^{m-1}+q^{2} e_{2} L^{m-2}+\ldots+(-q)^{m-1} e_{m-1} L+(-q)^{m} e_{m} I=0 .
$$

Note that the first proof of this identity in the algebras $\mathcal{L}(R, R)$ was given in GPS1.

Now, we pass to the second question. It is well known that if a Hecke symmetry $R$ comes from the quantum group $U_{q}(s l(N))$, the quantum determinant $\operatorname{det}_{\mathcal{L}(R, P)}(L)$ is central (see [FRT]). If in a given RTT algebra $\mathcal{L}(R, P)$ the quantum determinant is central, then, by imposing the condition $\operatorname{det}_{\mathcal{L}(R, P)}(L)=1$, we can define a Hopf algebra structure in the quotient algebra.

However, in general the quantum determinant is not central in the algebras $\mathcal{L}(R, P)$. As was shown in $\left[\mathrm{G}\right.$, the quantum determinant $\operatorname{det}_{\mathcal{L}(R, P)}(L)$ is central if and only if the matrix

$$
M=\left\|M_{i}^{j}\right\|, \quad \text { where } \quad M_{i}^{j}=u_{i i_{2} \ldots i_{m}} v^{i_{2} \ldots i_{m} j},
$$

\footnotetext{
${ }^{7}$ Emphasize, that if $F \neq R$ this property fails.
} 
is scalar.

Let us study the centrality of the quantum determinants in the algebras $\mathcal{L}(R, P)$, corresponding to the symmetries (2.6). By straightforward computations we get respectively the matrices $M$ for the symmetries from (2.6):

$$
-\frac{1}{2}\left(\begin{array}{cc}
q & 0 \\
0 & q^{-1}
\end{array}\right) \quad \text { and }-\frac{1}{2_{q}}\left(\begin{array}{ll}
1 & 0 \\
0 & 1
\end{array}\right) .
$$

Thus, the quantum determinant is not central in the algebra $\mathcal{L}(R, P)$, corresponding to the involutive symmetry from (2.6) and it is central in the algebra, corresponding to the Hecke symmetry from (2.6).

By contrast, in the $\mathrm{RE}$ algebras $\mathcal{L}(R, R)$ the quantum determinant is always central. Therefore, by imposing the condition $\operatorname{det}_{\mathcal{L}(R, R)}(L)=1$, we get a braided Hopf algebra structure in the quotient algebra (see GPS2]).

Now, we are going to discuss a way of reducing the quantum determinants to the so-called column-determinants and row-determinants, playing an important role in the theory of integrable systems.

We set $F=P$, i.e. consider the RTT algebra $\mathcal{L}(R, P)$. By using the relation (2.14) we get the following equality

$$
u_{i_{1} \ldots i_{m}} v^{j_{1} \ldots j_{m}} l_{j_{1}}^{k_{1}} \ldots l_{j_{m}}^{k_{m}}=u_{i_{1} \ldots i_{m}}\left\langle\mathrm{v}\left|L_{1} \ldots L_{m}\right| \mathrm{u}\right\rangle v^{k_{1} \ldots k_{m}} .
$$

Since the tensor $\mathrm{u} \not \equiv 0$, the factors $u_{i_{1} \ldots i_{m}}$ can be cancelled. Assume also, that $m=N$ and $v^{12 \ldots N}=1$. This condition can be satisfied by a proper normalization of $\mathrm{v}$, provided $v^{12 \ldots N} \neq 0$. Then we get

$$
\operatorname{det}_{\mathcal{L}(R, P)}(L)=v^{j_{1} \ldots j_{N}} l_{j_{1}}^{1} \ldots l_{j_{N}}^{N} .
$$

It is this form of the quantum determinant which is called the column-determinant of $L$. It is characterized by the property, that in each of its summands the multipliers $l_{i}^{j}$ are set in the order of columns of the matrix $L$ enumerated by the upper indices of $l_{i}^{j}$. Note that if $m \neq N$, we have no privileged component of the tensor $\mathrm{v}$ (like the component $v^{12 \ldots N}$ ).

In a similar way, if the multipliers in each of the summands of the determinant are set in the order of rows of the matrix $L$, we call it the row-determinant. If $m=N$ and $u_{12} \ldots N \neq 0$, we can transform the canonical determinant $\operatorname{det}_{\mathcal{L}(R, P)}$ to the form of row-determinant, which looks as follows

$$
\operatorname{det}_{\mathcal{L}(R, P)}(L)=l_{1}^{i_{1}} \ldots l_{N}^{i_{N}} u_{i_{1} \ldots i_{N}} .
$$

Observe that the column-determinant (resp., row-determinant) depends only on the tensor $\mathrm{v}$ (resp., u). Thus, if two symmetries have the same tensors $\mathrm{v}$ but different tensors $\mathrm{u}$ we have the identical form of the column-determinants but different row-determinants. It is just the case of the symmetries (2.6) considered above. We see that the column-determinants (the middle expressions in (2.8) and (2.10) ) are equal to each other but the row-determinants (the right expressions) are diffident.

Now, introduce the higher-dimensional counterparts of the symmetries (2.6) and exhibit the corresponding quantum determinants in the algebras $\mathcal{L}(R, P)$.

The Hecke symmetry $R$ coming from the QG $U_{q}(s l(N))$ is as follows

$$
R_{i j}^{k l}=q^{\delta_{k, l}} \delta_{j}^{k} \delta_{i}^{l}+\left(q-q^{-1}\right) \theta_{(l>k)} \delta_{i}^{k} \delta_{j}^{l},
$$

where $\theta_{(l>k)}=1$ if $l>k$ and $\theta_{(l>k)}=0$ if $l \leq k$.

Introduce, also, the involutive symmetry $R$ by its action on the basis vectors $x_{i} \otimes x_{j}$ of the space $V^{\otimes 2}$ :

$$
R\left(x_{i} \otimes x_{i}\right)=x_{i} \otimes x_{i}, \quad R\left(x_{i} \otimes x_{j}\right)=q x_{j} \otimes x_{i} \text { if } i<j, \quad R\left(x_{i} \otimes x_{j}\right)=q^{-1} x_{j} \otimes x_{i} \text { if } i>j .
$$


For the both symmetries the components of the tensors $u$ and $v$ are nontrivial iff their indices are pairwise distinct. Then for the both symmetries the nontrivial components of the tensor $\mathrm{v}$ can be taken as

$$
v^{j_{1} \ldots j_{N}}=(-q)^{l(\sigma)}
$$

where $l(\sigma)$ is the length (i.e. the minimal number of transpositions) of the permutation

$$
\sigma:(1 \ldots N) \mapsto\left(j_{1} \ldots j_{N}\right) .
$$

Sometimes, such a tensor $\mathrm{v}$ is called the $q$-Levi-Civita tensor.

As for the tensors $\mathrm{u}$ for these symmetries, they are respectively equal to

$$
u_{i_{1} \ldots i_{N}}=\alpha_{1}(-q)^{l(\sigma)}, \quad u_{i_{1} \ldots i_{N}}=\alpha_{2}\left(-q^{-1}\right)^{l(\sigma)},
$$

where

$$
\alpha_{1}^{-1}=q^{\frac{N(N-1)}{2}} N_{q} !, \quad \alpha_{2}^{-1}=N !
$$

are normalizing factors.

Similarly to the 2-dimensional example above we have for both symmetries the same "quantum coordinate ring"

$$
x_{i} x_{j}=q x_{j} x_{i}, \quad \forall i<j,
$$

often called the quantum torus (with the additional condition that the generators are invertible).

The formulae for the quantum column-determinants are also the same in both RTT algebras:

$$
\operatorname{det}_{\mathcal{L}(R, P)}(L)=\sum_{\sigma}(-q)^{l(\sigma)} l_{j_{1}}^{1} \ldots l_{j_{N}}^{N}
$$

In this form the quantum determinant $\operatorname{det}_{\mathcal{L}(R, P)}(L)$ is given in [FRT] for the RTT algebra associated with $U_{q}(s l(N))$ symmetry $R$.

However, the tensors $\mathrm{u}$ corresponding to the symmetries under consideration are different. Consequently, the row-determinants in the corresponding RTT algebras differ from each other.

In conclusion of the section, we turn to the so-called half-quantum algebras (HQA) and the corresponding quantum determinants.

Again, consider a compatible couple $(R, F)$, where $R$ is a skew-invertible Hecke symmetry. Introduce two systems of relations on the generating matrix $L=\left\|l_{j}^{i}\right\|_{1 \leq i, j \leq N}$ :

$$
\begin{array}{lll}
S^{(2)} L_{\overline{1}} L_{\overline{2}} A^{(2)}=0 & \Leftrightarrow & L_{\overline{1}} L_{\overline{2}} A^{(2)}=A^{(2)} L_{\overline{1}} L_{\overline{2}} A^{(2)}, \\
A^{(2)} L_{\overline{1}} L_{\overline{2}} S^{(2)}=0 & \Leftrightarrow & A^{(2)} L_{\overline{1}} L_{\overline{2}}=A^{(2)} L_{\overline{1}} L_{\overline{2}} A^{(2)},
\end{array}
$$

where $R$-symmetrizer $S^{(2)}$ and $R$-skew-symmetrizer $A^{(2)}$ are defined in (2.1). The matrices with overlined indices have the same meaning as above (see (2.2) ).

The following claim is well known and can be checked straightforwardly.

Proposition 5 The system (2.3) is equivalent to the union of the systems (3.5) and (3.6).

By imposing on generators only half of the relations (only (3.5) or (3.6)), we get a bigger algebra than that $\mathcal{L}(R, F)$. Nevertheless, even in such an algebra it is possible to develop some elements of linear algebra. We refer the reader to the paper [IO], where these algebras were introduced and studied. Some particular cases of these algebras were also considered in [CFR] and [CFRS] under the name of Manin matrices and $q$-Manin-matrices.

We call the right (resp., left) half-quantum algebra (HQA) a unital algebra defined by the system (3.5) (resp., (3.6) ). They will be respectively denoted $\mathcal{H}_{r}(R, F)$ and $\mathcal{H}_{l}(R, F)$. 
If $R$ is an even symmetry, we define the quantum determinant in the algebra $\mathcal{H}_{\epsilon}(R, F)$, $\epsilon \in\{r, l\}$ by formula (2.5) and denote it $\operatorname{det}_{\mathcal{H}_{\epsilon}(R, F)}(L)$.

Quantum elementary symmetric polynomials can be still defined in the algebras $\mathcal{H}_{\epsilon}(R, F)$ by formulae (2.13), where the projectors $A^{(k)}$ can be transferred to the rightmost position or put on the both positions: to the right and to the left of the chain of $L$-matrices..

Note that the quantum determinant in the algebra $\mathcal{H}_{\epsilon}(R, F)$ also differs from the highest quantum elementary symmetric polynomial by a factor.

Remark 6 Initially such type algebras were considered by Yu.Manin in [M]. Their definition is motivated by the following consideration. Let us endow the space $V$ with the coaction of an RTT algebra: $x_{i} \rightarrow t_{i}^{j} \otimes x_{j}$ and extend it to the space $V^{\otimes 2}$ by assuming that the generators $x_{i}$ and $t_{k}^{j}$ commute with each other. Then the relation (3.5) (resp., (3.6)), where we put $F=P$, means that the subspace $\operatorname{Im} A^{(2)}$ (resp., $\operatorname{Im} S^{(2)}$ ) is preserved under this coaction. However, if $F \neq P$, the assumption of commutativity of the generators $x_{i}$ and $t_{k}^{j}$ is not suitable any more.

Since the relation (2.14) is valid in any left HQA, the quantum determinant $\operatorname{det}_{\mathcal{H}(R, P)_{l}}(L)$, where $R$ is one of the symmetries (2.6) or its higher-dimensional counterpart, can be cast under the form of column-determinant. Whereas in any right HQA the quantum determinants can be cast under the form of row-determinant.

Nevertheless, in any HQA the number of relations between the generators is insufficient to prove that the elementary polynomials commute with each other.

\section{Generalized Yangians and integrable systems of CFRS type}

First, describe the Baxterization procedure which enables us to construct current braidings via involutive and Hecke symmetries (see [J, Jo $)$. Let us precise that by a current braiding $R(u, v)$ we mean an operator depending on parameters and subject to the braid relation of the following form:

$$
R_{12}(u, v) R_{23}(u, w) R_{12}(v, w)=R_{23}(v, w) R_{12}(u, w) R_{23}(u, v) .
$$

Given an involutive symmetry $R$, we associate with it a current braiding by the rule

$$
R(u, v)=R-\frac{I}{u-v}
$$

whereas for a Hecke symmetry $R$ the corresponding current braiding reads

$$
R(u, v)=R-\frac{\left(q-q^{-1}\right) u I}{u-v} .
$$

By a straightforward calculation one can verify that these operators do satisfy the relation (4.1). The current braidings (4.2) and (4.3) (and all corresponding algebras) will be respectively called the rational and trigonometric ones.

Introduce a countable set of elements $l_{j}^{i}[k], k \in \mathbb{Z}_{\geq 0}, 1 \leq i, j \leq N$, and consider a formal power series

$$
L(u)=\sum_{k \geq 0} L[k] u^{-k}, \quad L[k]=\left\|l_{i}^{j}[k]\right\|_{1 \leq i, j \leq N},
$$

that is $L(u)$ is an $N \times N$ matrix and its entries are power series in $u^{-1}$ with coefficients $l_{j}^{i}[k]$.

A generalized Yangian $\mathbf{Y}(R, F)$ is an associative unital algebra generated by elements $l_{j}^{i}[k]$ subject to the system

$$
R_{12}(u, v) L_{\overline{1}}(u) L_{\overline{2}}(v)-L_{\overline{1}}(v) L_{\overline{2}}(u) R_{12}(u, v)=0,
$$


where $L_{\overline{1}}(u)=L_{1}(u)$ and $L_{\overline{2}}(u)=F_{12} L_{\overline{1}}(u) F_{12}^{-1}$. Note that expanding the current matrix $L(u)$ is a series as indicated in (4.4), we get a countable set of polynomial relations on the generators $l_{i}^{j}[k]$.

In the case $F=R$ the algebra $\mathbf{Y}(R, R)$ with a supplementary condition $L[0]=I$ is called a braided or generalized Yangian of RE type (see [GS1] for detail). Note that the condition $L[0]=I$ is motivated by the evaluation morphism, similar to that in the Drinfeld's Yangian $\mathbf{Y}(g l(N))$. Note that the Drinfeld's Yangian is a particular case of the rational Yangians, corresponding to the symmetry $R=F=P$.

Observe that for a special value of the ratio $u / v=q^{2}$ in the trigonometric case the system (4.5) can be treated in terms of the HQA. A similar treatment is possible in the rational case if $u-v=1$.

More precisely, for the indicated relations between the parameters $u$ and $v$, the current braiding $R(u, v)$ becomes equal (up to a numerical factor) to the $R$-skew-symmetrizer $A^{(2)}$. Thus, in the rational (respectively, trigonometric) case we arrive at the relations

$$
\begin{aligned}
& A^{(2)} L_{\overline{1}}(u) L_{\overline{2}}(u-1)=L_{\overline{1}}(u-1) L_{\overline{2}}(u) A^{(2)}, \\
& A^{(2)} L_{\overline{1}}(u) L_{\overline{2}}\left(q^{-2} u\right)=L_{\overline{1}}\left(q^{-2} u\right) L_{\overline{2}}(u) A^{(2)} .
\end{aligned}
$$

Consequently, we have

$$
A^{(2)} L_{\overline{1}}(u) L_{\overline{2}}(u-1) S^{(2)}=0, \quad S^{(2)} L_{\overline{1}}(u-1) L_{\overline{2}}(u) A^{(2)}=0,
$$

in the rational case or, respectively,

$$
A^{(2)} L_{\overline{1}}(u) L_{\overline{2}}\left(q^{-2} u\right) S^{(2)}=0, \quad S^{(2)} L_{\overline{1}}\left(q^{-2} u\right) L_{\overline{2}}(u) A^{(2)}=0
$$

in the trigonometric case.

Using the formal Taylor series expansions

$$
L(u-1)=e^{-\partial_{u}} L(u) e^{\partial_{u}}, \quad L\left(q^{-2} u\right)=q^{-2 u \partial_{u}} L(u) q^{2 u \partial_{u}},
$$

where $\partial_{u}=\frac{d}{d u}$, we can rewrite the above relations in the form

$$
A^{(2)}\left(e^{-\partial_{u}} L_{\overline{1}}(u)\right)\left(e^{-\partial_{u}} L_{\overline{2}}(u)\right) S^{(2)}=0, \quad S^{(2)}\left(e^{\partial_{u}} L_{\overline{1}}(u)\right)\left(e^{\partial_{u}} L_{\overline{2}}(u)\right) A^{(2)}=0
$$

in the rational case and

$$
A^{(2)}\left(q^{-2 u \partial_{u}} L_{\overline{1}}(u)\right)\left(q^{-2 u \partial_{u}} L_{\overline{2}}(u)\right) S^{(2)}=0, \quad S^{(2)}\left(q^{2 u \partial_{u}} L_{\overline{1}}(u)\right)\left(q^{2 u \partial_{u}} L_{\overline{2}}(u)\right) A^{(2)}=0
$$

in the trigonometric case.

Thus, the operator $e^{-\partial_{u}} L(u)$ (respectively, $q^{-2 u \partial_{u}} L(u)$ ) plays the role of the generating matrix of a left HQA, whereas the operator $e^{\partial_{u}} L(u)$ (respectively, $q^{2 u \partial_{u}} L(u)$ ) plays the role of the generating matrix of a right HQA.

Now, define the quantum elementary symmetric polynomials in respectively rational and trigonometric cases as follows

$$
\begin{aligned}
& e_{0}(u)=1, \quad e_{k}(u)=\operatorname{Tr}_{R(1 \ldots k)} A^{(k)} L_{\overline{1}}(u) L_{\overline{2}}(u-1) \ldots L_{\bar{k}}(u-k+1), \quad k \geq 1, \\
& e_{0}(u)=1, \quad e_{k}(u)=\operatorname{Tr}_{R(1 \ldots k)} A^{(k)} L_{\overline{1}}(u) L_{\overline{2}}\left(q^{-2} u\right) \ldots L_{\bar{k}}\left(q^{-2(k-1)} u\right), \quad k \geq 1 .
\end{aligned}
$$

Observe that in these formulae the projectors $A^{(k)}$ can be put after the chain of the matrices $L$ or at two positions: to the right and to the left of the chain of the matrices $L$. Besides, the 
parameters of the matrices can be written in the inverse order. All these transformations lead to identical results.

Let $R$ to be an even symmetry of rank $m$ and define the quantum determinants in the generalized Yangians (respectively, rational and trigonometric) by setting

$$
\begin{aligned}
& \operatorname{det}_{\mathbf{Y}(R, F)}(L(u))=\left\langle\mathrm{v}\left|L_{\overline{1}}(u) L_{\overline{2}}(u-1) \ldots L_{\bar{m}}(u-m+1)\right| \mathrm{u}\right\rangle, \\
& \operatorname{det}_{\mathbf{Y}(R, F)}(L(u))=\left\langle\mathrm{v}\left|L_{\overline{1}}(u) L_{\overline{2}}\left(q^{-2} u\right) \ldots L_{\bar{m}}\left(q^{-2(m-1)} u\right)\right| \mathrm{u}\right\rangle .
\end{aligned}
$$

Note, that the quantum determinant equals to the highest elementary symmetric polynomial $e_{m}(u)$ up to a factor in full analogy with (2.15). If $F=P$ this equality is exact.

Consider the case $F=P$ in more detail. Assuming that $m=N$ and that $v^{1 \ldots N}$ and $u_{1 \ldots N}$ are non-trivial, we can cast the quantum determinant under the form of the column-determinant or the row-determinant:

$$
\operatorname{det}_{\mathbf{Y}(R, P)}(L)=v^{j_{1} \ldots j_{N}} l_{j_{1}}^{1}(u) \ldots l_{j_{N}}^{N}(u-N+1)=u_{i_{1} \ldots i_{N}} l_{1}^{i_{1}}(u-N+1) \ldots l_{N}^{i_{N}}(u)
$$

in the rational case and

$$
\operatorname{det}_{\mathbf{Y}(R, P)}(L)=v^{j_{1} \ldots j_{N}} l_{j_{1}}^{1}(u) \ldots l_{j_{N}}^{N}\left(q^{-2(N-1)} u\right)=u_{i_{1} \ldots i_{N}} l_{1}^{i_{1}}\left(q^{-2(N-1)} u\right) \ldots l_{N}^{i_{N}}(u)
$$

in the trigonometric one.

Now, let us take as $R$ the first (involutive) symmetry from (2.6) or its higher-dimensional counterpart. Since the corresponding generalized Yangian is rational, we can write the corresponding quantum determinant as follows

$\operatorname{det}_{\mathbf{Y}(R, P)}(L)=\sum_{\sigma}(-q)^{l(\sigma)} l_{\sigma(1)}^{1}(u) \ldots l_{\sigma(N)}^{N}(u-N+1)=\sum_{\sigma}\left(-q^{-1}\right)^{l(\sigma)} l_{1}^{\sigma(1)}(u-N+1) \ldots l_{N}^{\sigma(N)}(u)$.

In the case of the $U_{q}(s l(N))$ symmetries $R$, the corresponding generalized Yangian is trigonometric. Consequently, we have

$$
\operatorname{det}_{\mathbf{Y}(R, P)}(L)=\sum_{\sigma}(-q)^{l(\sigma)} l_{\sigma(1)}^{1}(u) \ldots l_{\sigma(N)}^{N}\left(q^{-2(N-1)} u\right)=\sum_{\sigma}(-q)^{l(\sigma)} l_{1}^{\sigma(1)}\left(q^{-2(N-1)} u\right) \ldots l_{N}^{\sigma(N)}(u) .
$$

We point out that the order of arguments in the matrices are opposite in the expressions for the column-determinant and for the row-determinant.

Also, note that similarly to the case of the algebras $\mathcal{L}(R, F)$, the quantum determinant is always central in the generalized Yangians of RE type $\mathbf{Y}(R, R)$ but it is not so in these of RTT type $\mathbf{Y}(R, P)$. More precisely, the quantum determinant $\operatorname{det}_{\mathbf{Y}(R, P)}(L)$ is central iff it is so for the quantum determinant $\operatorname{det}_{\mathcal{L}(R, P)}(L)$. This property is proved in [GS1].

The quantum versions of power sums can be also defined in all generalized Yangians and some quantum versions of the Cayley-Hamilton identity are valid in all of them.

The subalgebra generated in the generalized Yangian $\mathbf{Y}(R, F)$ by the quantum elementary polynomials is called the Bethe subalgebra and denoted $\mathcal{B}(R, F)$.

Proposition 7 [GSS] For any compatible couple $(R, F)$ of braidings such that $R$ is a skewinvertible involutive or Hecke symmetry the Bethe subalgebra $\mathcal{B}(R, F) \subset \mathbf{Y}(R, F)$ is commutative.

A particular case of this claim, corresponding to $F=P$ and $R$ coming from the $\mathrm{QG} U_{q}(\operatorname{sl}(N))$ is proved in [CFRS]. (Note that the formula for the projectors $A^{(k)}$ should be taken as in (2.1) 
The generalized Yangians of the RE type have a very important property: they admit evaluation morphisms. These morphisms were constructed in [GS1]. Similarly to the evaluation map in the Drinfeld's Yangian $\mathbf{Y}(P, P)$ they have the form

$$
L(u) \mapsto 1+\frac{M}{u}
$$

both in the rational and trigonometric cases. However, the target algebras, generating by matrix elements of $M$, are different: for the rational generalized Yangians it is a modified RE algebra, respective to the symmetriy $R$, and in the trigonometric case, it is a non-modified RE algebra.

In conclusion, we make a short remark. As we have already noticed, it is not possible to prove the commutativity of the quantum elementary polynomials in a HQA. However, the relations (4.6) and (4.7) enable one to establish this property in the generalized Yangians because they are more restrictive than the defining relations in the HQA.

\section{References}

[CFR] A.Chervov, G.Falqui, V.Rubtsov Algebraic properties of Manin matrices I, Advances in Applied Mathematics 43 (2009) 239-315.

[CFRS] A.Chervov, G.Falqui, V.Rubtsov, A.Silantyev Algebraic properties of Manin matrices II: q-analogues and integrable systems, Advances in Applied Mathematics 60 (2014) $25-89$.

[FRT] L.Faddeev, N.Reshetikhin, L.Takhtadzhyan Quantization of Lie groups and Lie algebras, Leningrad Math. J. 1 (1989) 193-225.

[G] D.Gurevich Algebraic aspects of quantum Yang-Baxter equation, Leningrad Math. J. 2 (1990) 119-148.

[GPS1] D.Gurevich, P.Pyatov, P.Saponov Hecke Symmetries and Characteristic Relations on Reflextion Equation Algebras, Lett. Math. Phys. 41 (1997) 255-264.

[GPS2] D.Gurevich, P.Pyatov, P.Saponov Representation theory of (modified) reflection equation algebra of $G L(m \mid n)$ type St. Petersburg Math. J. 20 (2008) 213-253.

[GS1] D.Gurevich, P.Saponov Braided Yangians, Journal of Geometry and Physics 138 (2019) $124-143$.

[GS2] D.Gurevich, P.Saponov From Reflection Equation Algebra to Braided Yangians, Recent Developements in Integrable Systems and Related Topics of Mathematical Physics, Kezenoi-Am, Russia, 2016, Springer Proceedings in Mathematics and Statistics.

[GSS] D.Gurevich, P.Saponov, A.Slinkin Bethe subalgebras in Braided Yangians and Gaudintype models, Comm. in Math. Physics 374 (2020) 689-704

[Gy] A.Gyoja A q-analogue of Young symmetrizers, Osaka J.Math. 23 (1986) 841-852.

[IO] A.Isaev, O.Ogievetsky Half-Quantum linear algebra, Nankai Series in Pure, Applied Mathematics and Theoretical Physics Symmetries and Groups in Contemporary Physics (2013) 479-486.

[IOP1] A.Isaev, O.Ogievetsky, P.Pyatov On quantum matrix algebras satisfying the CayleyHamilton-Newton identities, J. Phys. A 32 (1999) L115-L121. 
[IOP2] A.Isaev, O.Ogievetsky, P.Pyatov q-Multilinesr Algebra in Proc. of the Third International Workshop "Lie Theory and its applications in Physics III", Clausthal, Germany, 11-14 July 1999, Eds. H.-D. Doebner, V.K.Dobrev and J.Hilgert, World Sci. 2000, pp.268-279.

[J] M.Jimbo A q-difference Analog of $U(\mathfrak{g})$ and the Yang-Baxer Equation, Lett. Math. Phys. 10 (1985) 63-69.

[Jo] V.Jones Baxterization, Internat. J. Modern Phys. A 6 (1991) 2035-2043.

[KS] P.Kulish, E.Sklyanin Quantum spectral transform method recent developments, Integrable Quantum Field Theories, Proceedings of the Symposium Held at Tvaerminne, Finland, 23-27 March, 1981.

[L1] V.Lyubashenko Hopf algebras and vector-symmetries, (Russian) Uspekhi Mat. Nauk 41 (1986) 185-186.

[L2] V.Lyubashenko Thesis "Super-analysis and solutions to the equation of triangles", Kiev 1986 (Russian).

[M] Yu.Manin Quantum groups and noncommutative geometry, CRM, Montreal University, Montreal, Quebec 1988. 